\title{
Phenotypic and Genotypic Characterization of Enterococci from Clinical Isolates in a Tertiary Care Hospital
}

\author{
Mohamadiya Rizwana*, S. Parvathi and B. Appalaraju \\ Department of Microbiology, PSG IMSandR, Coimbatore, Tamil Nadu, India \\ *Corresponding author
}

\section{Keyw ords \\ Enterococci, Speciation, Antibiotic resistance, High level gentamicin, Vancomycin, Bifunctional gene. \\ Article Info \\ Accepted: \\ 17 June 2017 \\ Available Online: \\ 10 July 2017}

\author{
A B S T R A C T
}

Enterococci mainly being commensals in human faeces are now considered as an important cause of nosocomial infections. Of the infections, the commonly observed are urinary tract infections, abdominal infections followed by bacteremia, endocarditis and meningitis rarely. Speciation of Enterococcus aids in effective management of the infections caused by them. The initial treatment for enterococcal infections has become challenging due to development of resistance. The resistance has been observed mainly against aminoglycosides due to the presence of bi-functional gene. VRE strains are mostly isolated from patients with recurrent bacteremia, endovascular infections leading to increase deaths in the patients. To isolate and speciate enterococcal isolates from clinical samples, study the antibiotic susceptibility pattern and the genotype associated with the aminoglycoside and vancomycin resistance. Enterococcus isolates obtained from various sections were characterized by conventional phenotypic methods. The antibiotic susceptibility was studied by disc diffusion method. The resistant strains were further confirmed by MIC using automated methods following which the genotypic analysis was done. The incidence of Enterococci was $2.5 \%$ in which around $86.4 \%$ were obtained as pure isolates. Among the species E. faecalis was found to be the maximum. The urinary isolates exhibited sensitivity of around $92.3 \%$ to nitrofurantoin and among the non urinary isolates the maximum sensitivity was for linezolid. The molecular study showed van A to be most common gene with vancomycin resistance (71.42\%), and bifunctional gene among the aminoglycoside resistance (96\%). Enterococcus faecalis was the commonest species isolated. The maximum number of isolates was obtained from urinary samples. The increase incidence of resistance gene to high level gentamicin could result in failure to the synergy treatment of gentamicin in combination with penicillin group of antibiotics. The emergence of vancomycin resistant strains among the clinical isolates makes treatment options more challenging.

\section{Introduction}

Enterococci are gram positive organisms having the property to grow at $6.5 \% \mathrm{NaCl}$ concentration, $\mathrm{pH}$ of 9.6 and hydrolyze bile esculin. Initially they were grouped under group D Streptococcus but later with the help of DNA studies they are characterized under the genus Enterococcus in 1960. They were considered as harmless pathogens formerly, but in the recent years have been emerging as the most important causative agents in nosocomial infections (Murray et al., 1990).

Enterococci being the commonest micro flora of both humans and animals share their source 
of origin. They have been isolated from variety foods including cheese, fish, sausages, beef and pork. Studies have been reported that Enterococci have been isolated from various clinical and environmental samples all over the world. Among the isolates obtained clinically it has been observed that Enterococcus faecalis has been the predominant species clinically (Lancefield et al., 1933; Cetinkaya et al., 2000).

Enterococcal infections have been referred to as tough, tenacious and troublesome infections (Marothi et al., 2005).In the past years there has been an increase in the prevalence of enterococcal infections in hospitals and of particular concern is the emergence of antimicrobial resistant strains. Being second commonest organism behind the abdominal and pelvic infections, third in causing blood stream infections, cases have also been reported in causing $\mathrm{CNS}$ and neonatal infections.

Reports have been confirmed with their association with clinical conditions including respiratory tract infections, osteomyelitis and cellulitis (Fischer et al., 2009). Enterococci were initially regarded as the disease causing agent in the early nineties. But now various reports have been established reporting them as the second commonest pathogen (Kuhn et al., 2003).

Species identification becomes essential for investigating outbreaks in case of nosocomial infections and also susceptibility pattern varies accordingly which is also helpful in clinical management of infections due these agents (Gordon et al., 1992).

Infections caused by enterococcal pathogens were initially being treated with the combined treatment of cell wall active agents as penicillins and aminoglycoside as the synergistic effect was effective. But now due to the development of antibiotic resistance as high level aminoglycoside resistance, beta lactamase production and also resistance to vancomycin have led to serious concern in management of these nosocomial pathogens (Mendiratta et al., 2008). Vancomycin resistant Enterococcus was initially reported in 1980s, after which there was a increase in the incidence of its spread. The main phenotypes associated with its spread are Van A, Van B and Van C. Van A and Van B are mainly associated with $E$. faecalis and $E$. faecium. Van $\mathrm{C}$ is noted in E. gallinarum and E. casseliflavus. Among the phenotypes Van $\mathrm{A}$ is predominantly associated in the resistant strains.

VRE strains are mostly isolated from patients with recurrent bacteremia, endovascular infections leading to increase deaths in the patients. Vancomycin resistance and penicillin resistance are often seen co-existing which makes the treatment harder (Murray et al., 1990).

Resistance development in Enterococcus against glycopeptide antibiotics gets transferred from them to other bacterial pathogens which include Staphylococcus aureus, Streptococci, Listeria monocytogenes (Gold et al., 1996).

Thus due to the recent emergence in association with significant clinical infections and development of resistance, Enterococci have gained importance. Studies are thus required especially in tertiary care hospitals for appropriate isolation, identification and speciating them.

Antibiograms should also be followed up in accordance to the species. Emergence of VRE needs to be checked and limit its spread in hospital environment. CDC also stresses the control of VRE in hospitals by educating the staff in detecting VRE early, reporting them and action plan taken promptly. 
Our study aims to isolate, speciate the Enterococcus in a simplified manner and also to study the drug sensitivity and resistance patterns among the isolates obtained.

\section{Materials and Methods}

The Institutional Human Ethical clearance was obtained before the commencement of the study.

\section{Type of study}

Experimental study

\section{Confidentiality}

Confidentiality of the reports were maintained

\section{Sample size estimation}

A total of 250 isolates of Enterococci obtained from outpatient and inpatient samples from blood section, miscellaneous and urine section were processed for the species identification, antimicrobial sensitivity followed by molecular study.

Specimen processing: Specimens from various sections including urine, pus and blood for cultures were collected and processed in the laboratory.

\section{Pus/wound swabs}

The swabs were streaked in blood agar and MacConkey agar and were incubated at $37^{\circ} \mathrm{C}$ for 24 hours.

\section{Urine}

A loopful of the uncentrifuged specimen was examined as a wet mount for the presence of pus cells and bacteria. One loopful of specimen was inoculated onto blood agar and Mac Conkey agar. Plates were incubated at $37^{0} \mathrm{C}$ for 24 hours.

\section{Growth in sheep blood agar}

Following overnight incubation of the samples, colonies of Enterococcus were seen as grey colonies of about $0.5-1 \mathrm{~mm}$ in diameter with alpha, beta or gamma hemolysis.

\section{Growth in Mac Conkey agar}

Colonies were about $0.5-1 \mathrm{~mm}$ in size with a smooth surface and convex margins, tiny magenta colored following 18-24 hour growth.

\section{Catalase test}

A drop of the catalase reagent $(30 \%$ hydrogen peroxide) was added to the slide.

The growth was further applied to it with an applicator stick. Appearance of bubbles was taken as positive test.

Enterococcal colonies showed a negative test due to the absence of bubbles.

\section{Gram stain}

Smears made from the colony showed Gram positive cocci arranged singly, in pairs or in short chains.

\section{Storage}

If the isolate was catalase negative, gram staining showing the presence of gram positive cocci in pairs or short chains and growth in MacConkey agar as tiny lactose fermenting colonies, they were sub cultured and pure growth was stocked in Robertson's cooked meat medium in tubes.

The tubes were sealed and stored at $4-8^{0} \mathrm{C}$ for 3-6 months. When the test was to be done they were further sub cultured. 


\section{Identification tests}

\section{Growth in media with $\mathrm{pH}-\mathbf{9 . 6}$}

Isolated colonies of Enterococcus was streaked in a media with a $\mathrm{pH} 9.6$ and incubated at $37^{\circ} \mathrm{C}$ overnight. The presence of growth was taken as positive.

\section{Tolerance with $6.5 \% \mathrm{NaCl}$}

The colonies were inoculated in a broth with $6.5 \% \mathrm{NaCl}$, and incubated overnight. The next day the tubes were checked for any increase in turbidity. This was further confirmed by sub culturing onto the agar plates. Growth confirmed the presence of organism. Whereas absence of turbidity indicated organism was unable to grow in this concentration.

\section{Heat tolerance test}

Enterococcus was inoculated in Todd-Hewitt broth. It was later incubated in water bath set at $45^{\circ} \mathrm{C}$ overnight and $60^{\circ} \mathrm{C}$ for half an hour, and then sub cultured. Enterococci survived both the temperature showing positive reaction.

\section{Bile esculin test}

Colonies from 24 hour culture were inoculated in media containing $40 \%$ bile. Blackening of the medium confirmed positive reaction for Enterococci and non-Enterococci.

\section{PYR test}

The colonies were inoculated in PYR broth and incubated for a period of 4 hours. Later 3 drops of PYR reagent was added to it and change of colour to red was confirmed positive indicating Enterococci whereas absence of colour change indicates negative test.

\section{Motility test}

Stab culture of the colony was done in a semisolid media and motility was indicated by the spread of the organism into the media. The strains which did not diffuse into the media were considered non-motile. Tubes were incubated for up to 7 days for confirming motility.

\section{Arginine test}

A well isolated colony of Enterococcus from the culture was inoculated in Moeller's decarboxylase media with arginine as the amino acid and overlaid with mineral oil. After 24 hours of incubation the colour of the tube was changed to purple indicating a positive reaction. Yellow colour indicated only acid production only and no deamination.

\section{Potassium tellurite reduction test}

Colonies were streaked in media containing $0.004 \%$ potassium tellurite. E. faecalis formed black colonies with the reduction of tellurite to tellurium.

This test was used to differentiate E. faecalis from E. faecium.

\section{Pyruvate test}

When inoculated in a media containing pyruvate with bromothymol blue as indicator E. faecalis showed a colour change from green to yellow color after overnight incubation. Absence of colour change ruled out E. faecalis.

\section{Pigment production}

Ability to produce pigment was observed by a few species of Enterococci. This was tested by sub culturing colonies in nutrient agar. 
Any color produced was visible and also further confirmed by touching the colony with a sterile swab.

\section{Sugar fermentation tests}

Species identification was further done by sugar utilization by inoculating the organism in peptone with $1 \%$ sugars with Andrade indicator. The sugars used were mannitol, arabinose, raffinose, sorbose, sorbitol, lactose and sucrose. Any change in colour from colourless to pink indicated the positive sugar fermentation test. Absence of colour change was taken as negative.

\section{Antimicrobial susceptibility test: (CLSI 2015)}

The 250 test isolates of Enterococci were checked for the antimicrobial susceptibility pattern using Kirby-Bauer disc diffusion method. Three or four pure colonies were inoculated in peptone water and incubated for about 3 hours. The turbidity of the growth was matched to 1 of Mc Farlands. A lawn culture of the broth with a sterile swab was done in Muller Hinton agar and the discs were placed at a distance of $24 \mathrm{~mm}$.

About 6 discs were kept on each plate. The discs used were Penicillin (10U), Ampicillin

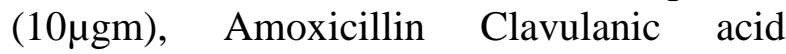

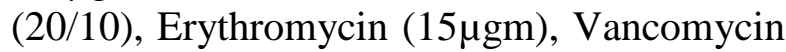
$(30 \mu \mathrm{gm})$, High level gentamicin $(120 \mu \mathrm{gm})$, Linezolid $(30 \mu \mathrm{gm})$, Tetracycline $(30 \mu \mathrm{gm})$. For isolates from urine section Norfloxacin $(10 \mu \mathrm{gm})$ and Nitrofurantoin $(300 \mu \mathrm{gm})$ was kept. All discs were bought from HIMEDIA.

Zones of inhibition were measured and recorded and the organism was interpreted as sensitive or resistant as per the recommendations from CLSI guidelines. The quality Control used was Enterococcus faecalis ATCC 29212.

\section{Screening for high level gentamicin} resistance (CLSI 2015)

Screening media for high level gentamicin resistance was prepared. Screening for high level gentamicin resistance was done by agar dilution method. BHI agar with gentamicin at a concentration of $500 \mu \mathrm{gm} / \mathrm{ml}$ was added and the plates were prepared. Inoculum of $10 \mu \mathrm{L}$ of $0.5 \mathrm{Mc}$ Farland was spot inoculated. Presence of even 1 colony was interpreted as a resistant strain. These strains were collected for molecular studies.

\section{Detection of beta lactamase (Marothi et al., 2005)}

The detection of beta lactamase test was done based on the chromogenic cephalosporin method. Nitrocefin disc was obtained commercially from BD. The disc was moistened with sterile saline and pure isolated colony was applied on it. The reaction was read within $5 \mathrm{~min}$. Isolates producing beta lactamase produced a red color.

\section{Molecular study of isolates}

A representative sample of 50 high level gentamicin resistant isolates was selected for genomic analysis. The DNA was extracted using the QIAGEN kit. Followed by extraction, the isolates were analysed for the presence of Bifunctional resistant geneaac (6') le aph (2") la. The vancomycin resistant isolates were checked for the presence of van A gene simultaneously.

\section{Detection of aac (6') le aph (2") la gene}

The primers for HLGR with a base pair of 348 bp were amplified. The sequence was obtained from the studies done previously ${ }^{11}$. PCR Master mix: The master mix was

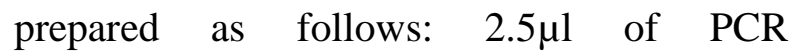
buffer, $2.5 \mu 1$ of $\mathrm{MgCl} 2,2.5 \mu 1$ of DNTPs, 
$10.2 \mu 1$ of Mili Q H2O, $1 \mu 1$ of forward and reverse primers and $0.3 \mu 1$ of Taq Polymerase was added to eppend off. From the above mixture $18 \mu \mathrm{l}$ was taken and $5 \mu \mathrm{l}$ of the extracted DNA was added.

Primers for aac (6')Ie- aph (2")la with base pair of 348 bp (Vakulenko et al., 2003):

F (5'CAGAGCCTTGGGAAGATGAAG3') R (5'CCTCGTGTAATTCATGTTCTGGC3')

\section{PCR program}

The program consisted of initial denaturation step at $95^{\circ} \mathrm{C}$ for 10 minutes followed by 30 cycles of denaturation at $94^{0} \mathrm{C}$ for 300 seconds, annealing at $56^{\circ} \mathrm{C}$ for 1 minute and extension at $72^{\circ} \mathrm{C}$ for a period of 1 minute. The program had a holding temperature of $72^{0} \mathrm{C}$ for 10 minutes.

\section{Analysis}

PCR products were analyzed by running gel electrophoresis with $1.5 \%$ agarose gel in Tris Boric acid buffer. The samples along with the controls obtained by running ladder were analysed.

The gel was stained with ethidium bromide and the bands were obtained were visualized under UV light and also in automated GEL DOC viewer.

\section{Detection of vanA gene}

The primers with a base pair of $732 \mathrm{bp}$ for detection of vanA were obtained from the studies done previously.

Primers for van A with base pair of 732bp (Dutka-Malen et al., 1995):

F (5' GGGAAAACGACAATTGC-3') R (5'GTACAATGCGGCCGTCGTTA-3')

\section{PCR program}

This program had a denaturation step at $95^{\circ} \mathrm{C}$ for 5 minutes followed by 30 cycles of DNA denaturation at $95^{\circ} \mathrm{C}$ for 1 minute, annealing at $54^{\circ} \mathrm{C}$ for 45 seconds and primer extension at $72^{\circ} \mathrm{C}$ for 45 seconds. This was followed by a holding temperature of $72^{\circ} \mathrm{C}$ for 10 minutes.

\section{Analysis}

PCR products were analyzed by running gel electrophoresis with $1.5 \%$ agarose gel in Tris Boric acid buffer. The samples along with the controls obtained by running ladder were analysed. The gel was stained with ethidium bromide and the bands were obtained were visualized under UV light and in automated GEL DOC viewer.

\section{Results and Discussion}

All the 250 isolates of Enterococcus from various clinical samples including urine, pus, wound swab, blood and CSF were processed.

The maximum number of isolates was obtained from urine section (184) followed by wound swabs (24), blood section (14), 4 from peritoneal samples, 1 from CSF (Figure 1). The majority of the isolates were obtained as pure growth (216) and 34 was obtained as mixed from the samples processed. Figure 2 depicts the speciation of enterococcal isolates in which E. faecalis was the predominant species (157), succeeded by E. faecium (79), E. raffinosus (7), and E. durans (5) and $E$. casseliflavus (2).

Illustration 1 shows the genus specific biochemical tests used in the identification.

The antibiotic susceptibility pattern of the enterococcal isolates by disc diffusion method revealed that out of the 250 isolates, about $69.2 \%$ were ampicillin sensitive, $49.2 \%$ were 
high level gentamicin sensitive, $89.3 \%$ were sensitive to erythromycin. The maximum number of isolates was found to be sensitive to vancomycin $(97.2 \%)$ in our study (Table 1). Among the urinary isolates maximum sensitivity was noticed to nitrofurantoin (92.3\%) (Table 2) and norfloxacin (83.1\%).

The maximum sensitivity was observed for linezolid followed by vancomycin, teicoplanin, ampicillin and high level gentamicin. Among the non-urinary isolates erythromycin sensitivity was observed to be the highest.

The chromogenic nitrocefin disc was used to screen the isolates for the presence of beta lactamase. In our study none of the isolates were found to be positive for beta lactamase.

Figure 3 shows the presence of Bifunctional gene aac (6')1eaph(2")la among the high level gentamicin resistant strains. Out of the 50 randomly selected resistant strains for genotype study, 48 had the gene.

Illustration 2 shows the presence of bands of size $348 \mathrm{bp}$ during the gel electrophoresis.

In our study 7 out of the 250 isolates were found to be resistant to vancomycin. The genotypic analysis was done among these resistant strains using the van A gene. The results showed that 5 of the 7 strains were found to be positive for van A gene (Figure 4).

Illustration 3 shows the presence of van A gene of base pair 732, confirming its presence among the resistant strains.

Enterococcus has been noted to cause a majority of urinary tract infections. This being the most common followed by bacteremia, sepsis and even case reports of meningitis has been reported. In our study out of 250 samples studied the majority of the isolates were obtained from urinary tract infections (73.6\%) followed by wound swabs $(9.6 \%)$ and blood $(5.6 \%)$. This correlates well with other studies in which Enterococci was obtained from urine isolates maximally (Karmarkar et al., 2004, Mathur et al., 2003). According to the studies Enterococci are considered as the commonest urinary pathogen justifying the increase in the rate of isolation from urinary samples (Murray et al., 1990).

Among the various species, in our study $E$. faecalis was the most common isolate accounting for $62.8 \%$ followed by E. faecium, E. durans, E. raffinosus and E. casseleflavus. Similar findings were reported from other studies done in Bangalore in which E.faecalis was $59 \%$, E. faecium was $38 \%$ (Golia et al., 2014).

Enterococci show intrinsic resistance to cotrimoxazole, clindamycin, cephalosporins and normal aminoglycosides. Thus these antibiotics are not tested (Mendiratta et al., 2008).

The antibiotic susceptibility pattern of the isolates in our study showed the following:

The sensitivity to linezolid was maximum (98.8\%) followed by vancomycin $(97.2 \%)$, erythromycin $(89.3 \%)$, teicoplanin $(75.2 \%)$, ampicillin (69.2\%), high level gentamicin $(49.2 \%)$ and penicillin $(25 \%)$.

Among the urinary isolates maximum sensitivity was noted in Nitrofurantoin (92.3\%) followed by Norfloxacin (83.15\%). Studies done in North India showed a similar susceptibility pattern with increased resistance to penicillin and aminoglycosides. Also the sensitivity percentage to linezolid and vancomycin was higher compared to other antibiotics (Mulla et al., 2012). 
Fig.1 Distribution of Enterococcus isolates among various samples ( $\mathrm{N}=250)$

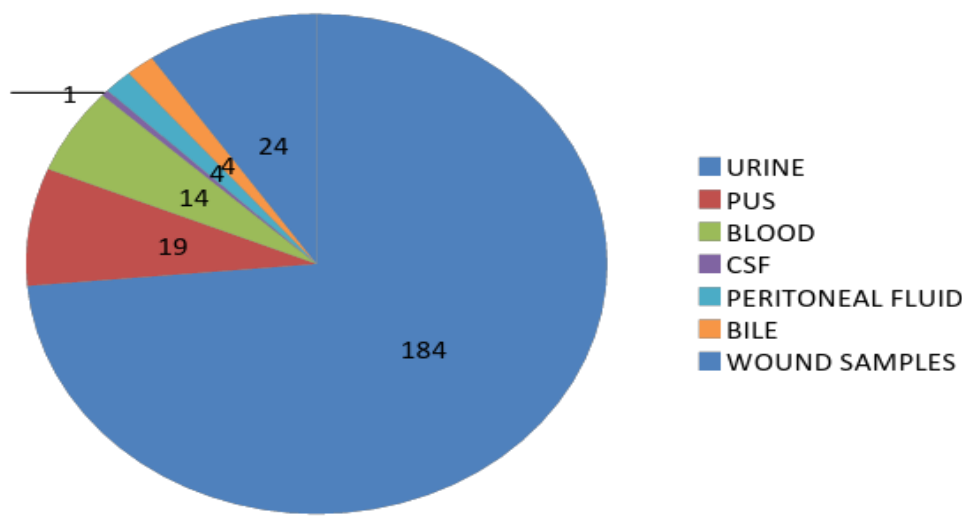

Fig.2 Speciation of Enterococcus from clinical isolates $\mathrm{N}=250$

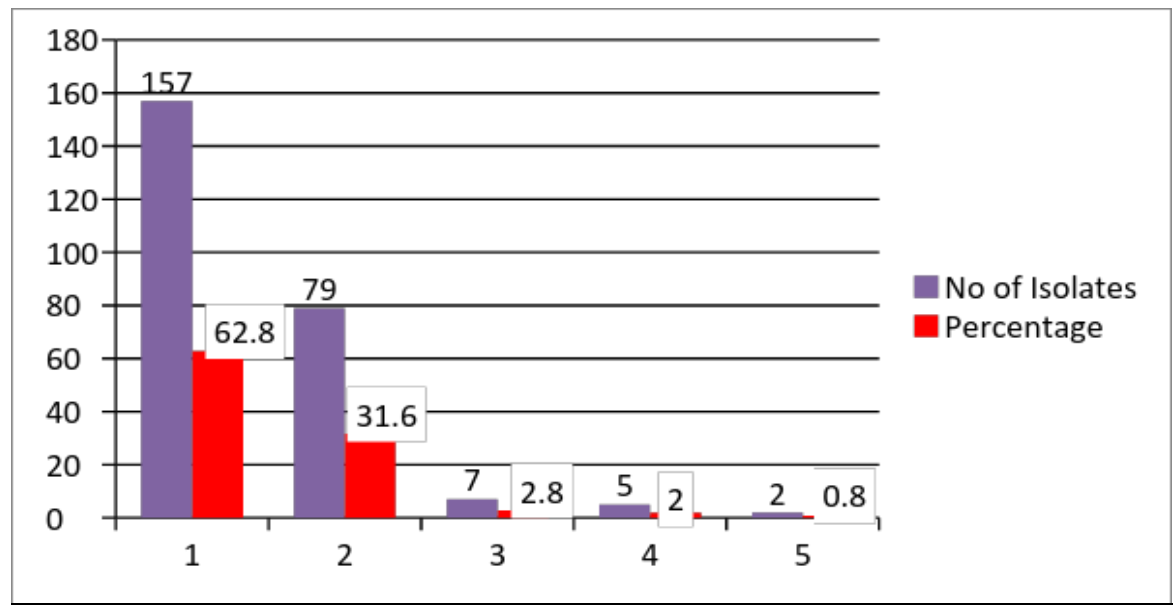

\begin{tabular}{|c|c|}
\hline E.faecalis & 1 \\
\hline E.faecium & 2 \\
\hline E.raffinosus & 3 \\
\hline E.durans & 4 \\
\hline E.casseliflavus & 5 \\
\hline
\end{tabular}

Fig.3 Detection of the presence of aac (6') le aph (2") la gene $\mathrm{N}=50$

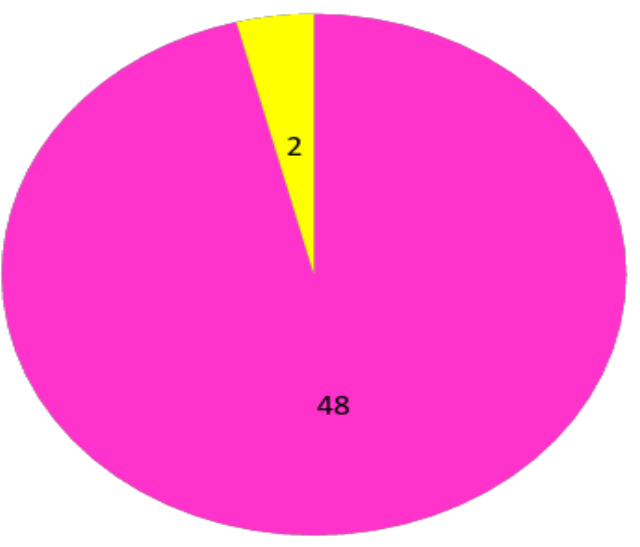


Fig.4 Detection of van A gene among the VRE isolates $(\mathrm{N}=7)$

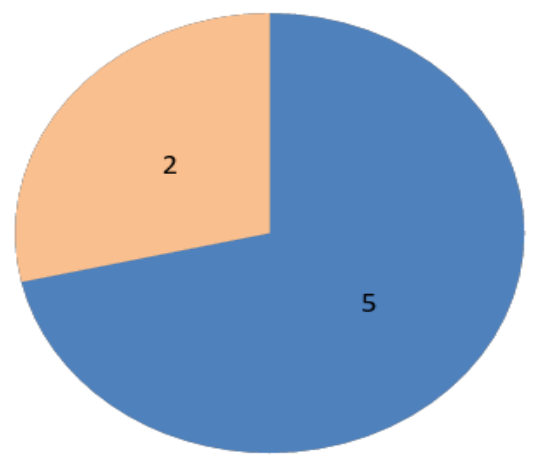

Present

$\square$ Absent

Table.1 Antibiotic susceptibility pattern of Enterococcal isolates

$\mathrm{N}=250$

\begin{tabular}{|l|l|l|}
\hline RESISTANCE & SENSITIVE & ANTIBIOTICS \\
\hline 77 & 173 & AMPICILLIN \\
\hline 30.8 & 69.2 & $\%$ \\
\hline 127 & 123 & HIGH LEVEL GENTAMICIN \\
\hline 50.8 & 49.2 & $\%$ \\
\hline 7 & 243 & VANCOMYCIN \\
\hline 2.8 & 97.2 & $\%$ \\
\hline 3 & 247 & LINEZOLID \\
\hline 1.2 & 98.8 & $\%$ \\
\hline 62 & 188 & TEICOPLANIN \\
\hline 24.8 & 75.2 & $\%$ \\
\hline
\end{tabular}

Table.2 For urinary isolates

\begin{tabular}{|l|l|l|}
\hline RESISTANCE & SENSITIVE & ANTIBIOTICS \\
\hline 31 & 153 & NORFLOX \\
\hline 16.85 & 83.15 & $\%$ \\
\hline 14 & 170 & NITROFURANTOIN \\
\hline 7.61 & 92.39 & $\%$ \\
\hline 37 & 147 & TETRACYCLINE \\
\hline 20.11 & 79.89 & $\%$ \\
\hline
\end{tabular}

Table.3 For non urinary isolates

\begin{tabular}{|c|c|c|}
\hline \multicolumn{2}{|c|}{$\mathrm{N}=66$} \\
\hline RESISTANCE & SENSITIVE & ANTIBIOTICS \\
\hline 49 & 17 & PENICILLIN \\
\hline 74.25 & 25.75 & $\%$ \\
\hline 7 & 59 & ERYTHROMYCIN \\
\hline 10.7 & 89.3 & $\%$ \\
\hline
\end{tabular}


Illustration.1 Genus specific test
A) Bile-Esculin Test
B) PYR Test
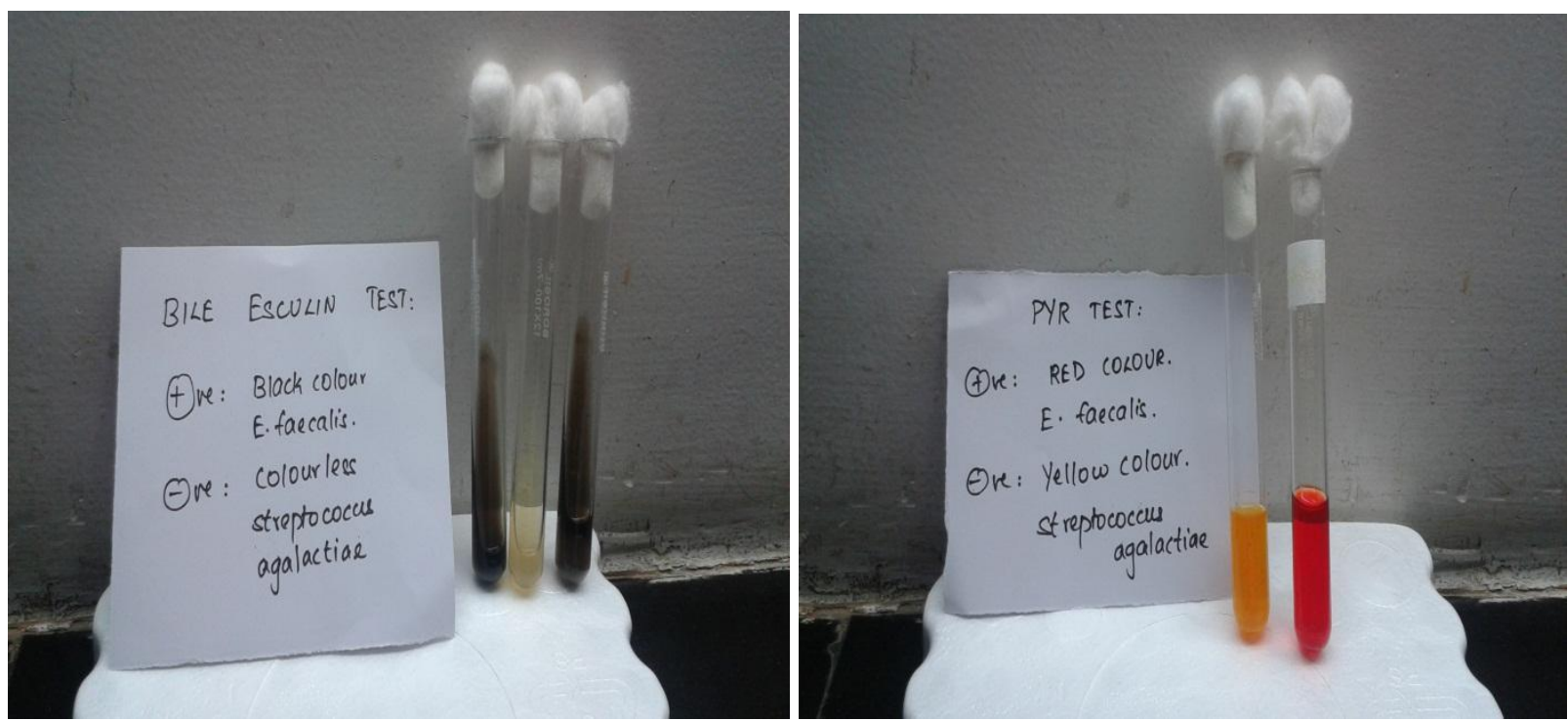

C) $6.5 \% \mathrm{NaCl}$ test

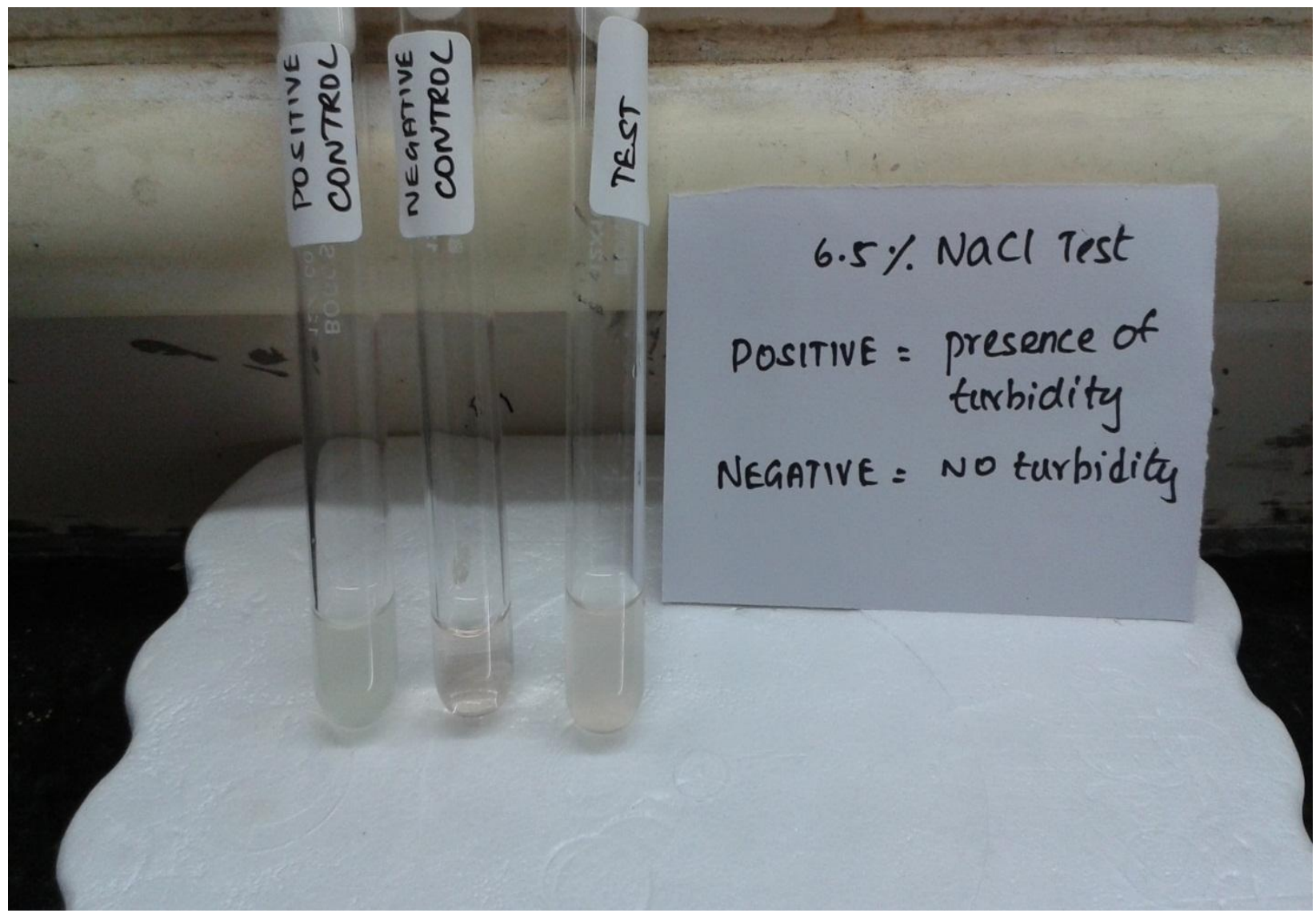


Illustration.2 PCR showing aac(6')-aph(2”) BAND OF SIZE 348 bp

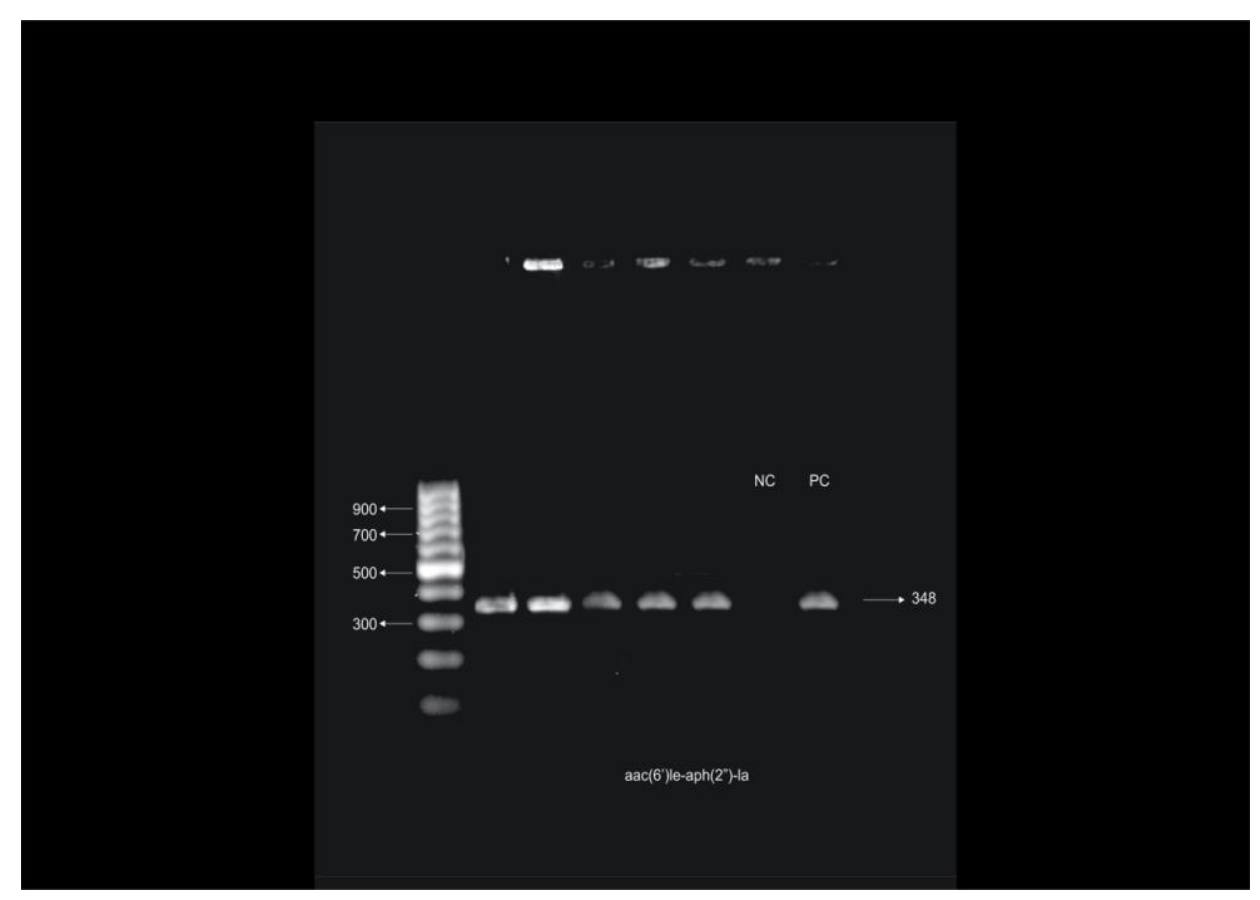

Illustration.3 PCR showing van A gene of band size 732bp

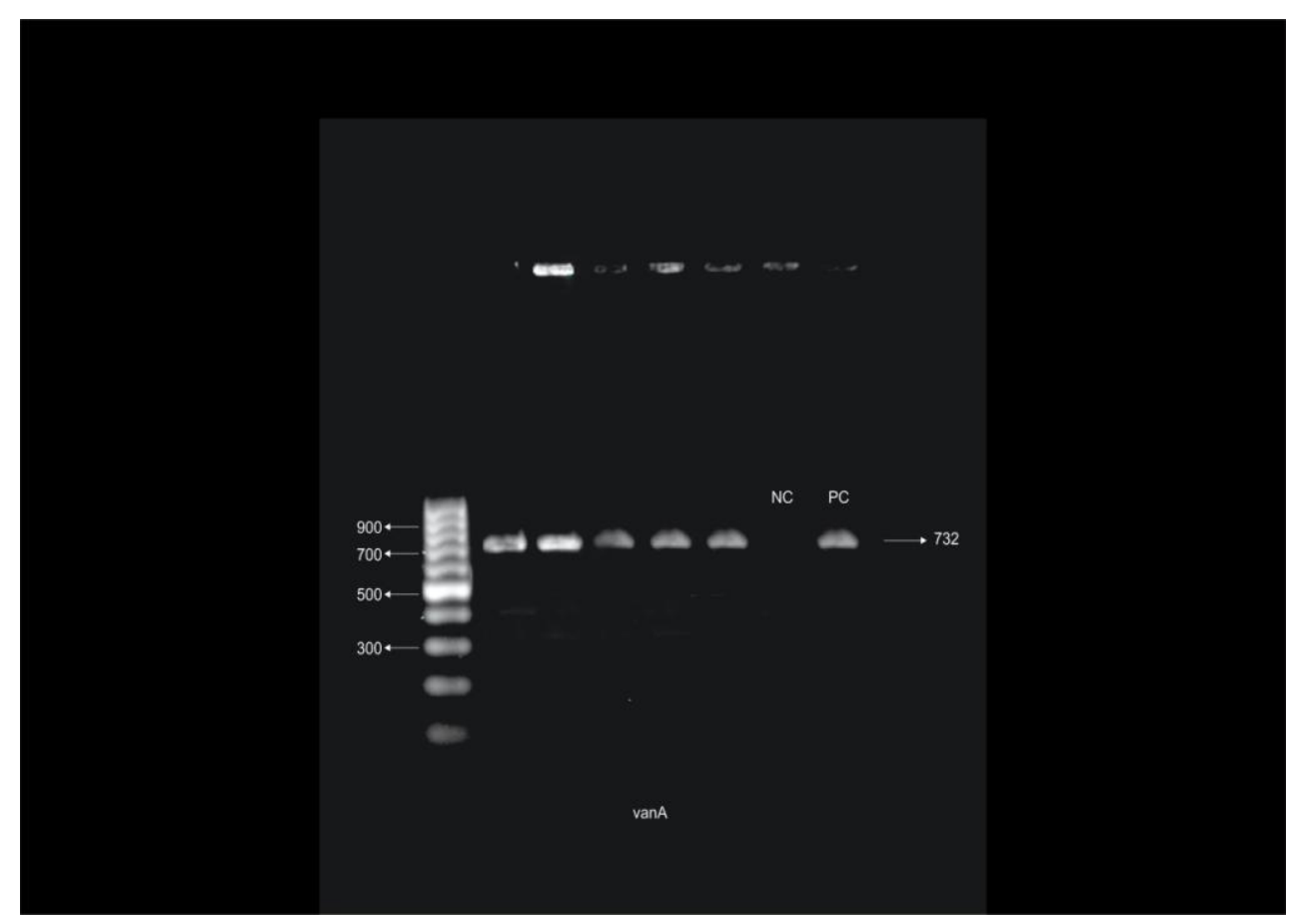

Another study showed the percentage of high level gentamicin resistance to be $68 \%$ (Randhawa et al., 2004). Reports from South
India showed the resistance percentage of high level aminoglycoside to be more than 50\% (Sreeja et al., 2012). 
The detection of beta lactamase production by the chromogenic nitrocefin disc method showed that none of the isolates had beta lactamase enzyme. Studies done in Delhi and in Maharashtra also showed similar results (Jain et al., 2011; Mendiratta et al., 2008). The resistance to beta lactams though common, the mechanism could be due to alteration in penicillin binding protein PBP 5 which is more common than beta lactamase production (Murray et al., 1992).

The molecular study done to detect the presence of the resistant gene confirmed the presence of the bifunctional gene aac (6') leaph (2") la of base pair 348 to be present in 48 $(96 \%)$ out of the 50 randomly selected resistant strains.

Studies done in Chennai have showed the presence of this gene in $38.2 \%$ of the isolates (Padmasini et al., 2014) whereas there are other studies in which all the resistant isolates possessed this bifunctional gene (Hasani et al., 2012). The resistance to high level gentamicin indicates resistance to all the aminoglycosides except streptomycin (CLSI 2015). The combination effect of aminoglycoside with beta lactam antibiotic is not helpful in the treatment of patients possessing such resistant strains.

Out of the 250 isolates the vancomycin resistant isolates obtained in our study was $2.8 \%$. Studies have shown the prevalence of vancomycin resistance to be less in India ranging around 1.7-20\% (Sreeja et al., 2012).

Molecular studies revealed the presence of van A gene in $5(71.42 \%)$ isolates. Studies have also been reported showing van $A$ to be the most common genotype isolated among the vancomycin resistant strains (Praharaj et al., 2013)

Out of the seven isolates, five were $E$. faecium and two were E. casseliflavus. The remaining two isolates were E. casseliflavus which would have exhibited intrinsic resistance to vancomycin with van $\mathrm{C}$ gene (Praharaj et al., 2013).

The incidence of Enterococci from the clinical isolates in our study was $2.5 \%$.

The incidence of the pure isolates was $86.4 \%$ in comparison to the mixed isolates which was found to be $13.6 \%$.

Among the isolates of Enterococci, the predominant species was E. faecalis $(62.8 \%)$ followed by E. faecium (31.6\%), E. raffinosus (2.8\%), E. durans (2\%) and E. casseliflavus $(0.8 \%)$.

The antibiotic sensitivity pattern of the urinary isolates showed $92.3 \%$ sensitive to nitrofurantoin and $83.15 \%$ sensitive to norfloxacin. Among the non-urinary isolates maximum sensitivity was observed in linezolid which was $98.8 \%$ followed by vancomycin (97.2\%), erythromycin (89.3\%), teicoplanin (75.2\%), ampicillin (69.2), high level gentamicin sensitivity (HLG) (49.2\%) and pencillin (25\%) (Table 3).

The vancomycin resistance was found to be $2.8 \%$ in E. faecium.

All the isolates were found to be beta lactamase negative by chromogenic (nitrocefin) method proving the alteration in PBP5 to be the more common mechanism involved in penicillin resistance.

The molecular characterization of HLGR(high level gentamicin resistant) strains, 50 isolates selected randomly out of 127 , showed the presence of bifunctional gene aac(6')le-aph(2")la to be around $96 \%$. (Primers of base pair 348 - F (5'CAGAGCCTTGGGAAGATGAAG3') R (5'CCTCGTGTAATTCATGTTCTGGC3') 
The presence of van A gene was found to be $71.42 \%$ in our study.

\section{Acknowledgements}

I thank Dr. S. Ramalingam and Dr. B. Appalaraju for permitting me to carry out the work in the Department of Microbiology, PSGIMSandR, Coimbatore. I acknowledge the kind help rendered by the supervisor Dr. Parvathi.S. for having guided at every level.

\section{References}

Cetinkaya et al., (2000) Vancomycin-resistant Enterococci. Clinical microbiology reviews 13, no: 686-707.

CLSI. Clinical and Laboratory Standards Institute. Performance Standards for antimicrobial susceptibility testing. CLSI document M100-524.Wayne, PA: Clinical and Laboratory standard Institute 2014.

Dutka-Malen et al., (1995) "Detection of glycopeptide resistance genotypes and identification to the species level of clinically relevant Enterococci by PCR." Journal of clinical microbiology 33, no. 1: 24-27.

Fisher et al., (2009) "The ecology, epidemiology and virulence of Enterococcus." Microbiology 155, no. 6: 1749-1757.

Gold et al., (1996). "Antimicrobial-drug resistance. "New England Journal of Medicine 335, no. 19:1445-1453.

Golia et al., (2014) "Isolation and speciation of Enterococci from various clinical samples and their antimicrobial susceptibility pattern with special reference to high level aminoglycoside resistance. "International Journal of Medical Research and Health Sciences 3, no. 3: 526-529.

Gordon et al., (1992) "Antimicrobial susceptibility patterns of common and unusual species of Enterococci causing infections in the United States. Enterococcal Study Group." Journal of clinical microbiology 30, no. 9: 23732378.

Hasani et al., (2012). "Molecular screening of virulence genes in high-level gentamicin-resistant Enterococcus faecalis and Enterococcus faecium isolated from clinical specimens in Northwest Iran." Indian journal of medical microbiology 30, no. 2: 175.

Jain et al., (2011) "Clinico-epidemiological profile and high-level aminoglycoside resistance in enterococcal septicemia from a tertiary care hospital in east Delhi. "International Journal of Applied and Basic Medical Research 1, no. 2: 80 .

Karmarkar et al., (2004) "Enterococcal infections with special reference to phenotypic characterization and drug resistance." Indian Journal of Medical Research 119: 22-25.

Kühn et al., (2003) "Comparison of enterococcal populations in animals, humans, and the environment-a European study. "International journal of food microbiology 88, no. 2: 133145.

Lancefield et al., (1930). "A serological differentiation of human and other groups of hemolytic streptococci." The Journal of experimental medicine 57, no. 4: 571-595.

Marothi et al., (2005) "Enterococcal resistance-an overview." Indian journal of medical microbiology 23, no. 4: 214.

Mathur et al., (2003) "Antimicrobial resistance in Enterococcus faecalis at a tertiary care centre of northern India." Indian Journal of Medical Research 118: 25-28.

Mendiratta et al., (2008). "Status of high level aminoglycoside resistant Enterococcus faecium and Enterococcus faecalis in a 
rural hospital of central India." Indian Journal of Medical Microbiology 26, no. $4: 369$.

Mulla et al., (2012)"Prevalence of Enterococci with higher resistance level in a tertiary care hospital: a matter of concern." therapy 1, no. 2: 3 .

Murray et al., (1992). "Beta-lactamaseproducing Enterococci." Antimicrobial agents and chemotherapy 36, no. 11: 2355.

Murray et al., (1990)"The life and times of the Enterococcus." Clinical microbiology reviews 3, no.1: 46-65.

Padmasini et al., (2014) "High level aminoglycoside resistance and distribution of aminoglycoside resistant genes among clinical isolates of Enterococcus species in Chennai, India." The Scientific World Journal 2014.

Praharaj et al., (2013) "Phenotypic and genotypic characterization of vancomycin resistant Enterococcus isolates from clinical specimens." The Indian journal of medical research 138 , no. 4: 549.

Randhawa et al., (2004) "Aminoglycoside resistance in Enterococci isolated from paediatric septicaemia in a tertiary care hospital in north India." Indian Journal of Medical Research 119: 77-79.

Sreeja et al., (2012). "The prevalence and the characterization of the Enterococcus species from various clinical samples in a tertiary care hospital." Journal of clinical and diagnostic research: JCDR 6, no. 9: 1486.

Vakulenko et al., (2003) "Multiplex PCR for detection of aminoglycoside resistance genes in Enterococci." Antimicrobial agents and chemotherapy 47, no. 4: $1423-1426$.

\section{How to cite this article:}

Mohamadiya Rizwana, S. Parvathi and Appalaraju, B. 2017. Phenotypic and Genotypic Characterization of Enterococci from Clinical Isolates in a Tertiary Care Hospital. Int.J.Curr.Microbiol.App.Sci. 6(7): 1160-1173. doi: https://doi.org/10.20546/ijcmas.2017.607.141 